\title{
Intraoperative transit-time flow measurement and high-frequency ultrasound assessment in coronary artery bypass grafting
}

David P. Taggart, MD, PhD, ${ }^{a}$ Daniel J. F. M. Thuijs, MD, ${ }^{b}$ Gabriele Di Giammarco, MD,

John D. Puskas, MD, ${ }^{\mathrm{d}}$ Daniel Wendt, MD, PhD,${ }^{e}$ Gregory D. Trachiotis, MD, ${ }^{\mathrm{f}}$

Teresa M. Kieser, MD, PhD, ${ }^{g}$ A. Pieter Kappetein, MD, PhD, ${ }^{b}$ and Stuart J. Head, MD, PhD ${ }^{b}$

\section{ABSTRACT}

Objectives: We evaluated the influence of transit-time flow measurement with epicardial and epiaortic high-frequency ultrasound in patients undergoing coronary artery bypass grafting procedure.

Methods: The Registry for Quality Assessment with Ultrasound Imaging and Transit-time Flow Measurement in Cardiac Bypass Surgery study is a multicenter, prospective study among 7 international centers performing coronary artery bypass grafting procedures. The primary end point was any change in the planned surgical procedure. Major secondary end points consisted of the rate and reason for surgical changes related to the aorta, in situ conduits, coronary targets, and completed grafts, and the rate of in-hospital mortality and major morbidity.

Results: Between April 2015 and December 2017, 1046 patients were enrolled. Of those, 1016 were included in the final analyses. Mean age was 65.9 years, $14.0 \%$ were women, and diabetes was present in $39.6 \%$. Off-pump procedures were performed in 39.6\% and bilateral internal thoracic arteries in 30.5\%. The primary end point occurred in $25.2 \%$ of patients $(n=256)$ and in $77 \%$ (197 out of 256) this was based on transit-time flow measurement and/or high-frequency ultrasound. Surgical changes were related to the aorta in $9.9 \%$, to in situ conduits in $2.7 \%$, and the coronary targets in $22.6 \%$. Graft revision occurred in $7.8 \%$, including revisions of the proximal and/or distal anastomosis in $6.6 \%$. In-hospital adverse event rates were $0.6 \%$ for mortality, $1.0 \%$ for cerebrovascular events, and $0.3 \%$ for myocardial infarction.

Conclusions: Surgical changes related to the aorta, conduits, coronary targets, and anastomosis were made in $25 \%$ of patients. This was associated with low operative mortality and low major morbidity. Transit-time flow measurement and high-frequency ultrasound may improve the quality, safety, and efficacy of coronary artery bypass grafting procedures and should be considered as a routine procedural aspect. (J Thorac Cardiovasc Surg 2020;159:1283-92)

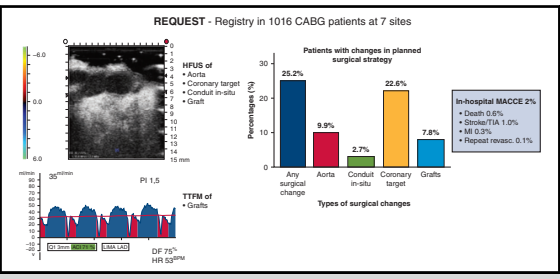

Changes to the planned surgical strategy were made in $25.2 \%$ of cases with few complications.

\section{Central Message}

In 7 international centers routinely performing CABG, $25.2 \%$ of patients required a change in surgical strategy that was accompanied by low in-hospital mortality and morbidity.

\section{Perspective}

Based on the results from the multicenter, prospective REQUEST study, TTFM and HFUS may improve the quality, safety, and efficacy of CABG procedures, and should therefore be considered as routine intraoperative quality control measures during CABG procedures.

See Commentaries on pages 1293 and 1295.

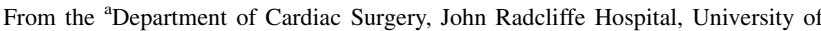
Oxford, Oxford, United Kingdom; ' ${ }^{\mathrm{b}}$ Department of Cardiothoracic Surgery, Erasmus University Medical Centre, Rotterdam, The Netherlands; ${ }^{c}$ Department of Cardiac Surgery, Università degli Studi "G. D'Annunzio" Chieti-Pescara, Chieti, Italy; ${ }^{\mathrm{d}}$ Department of Cardiovascular Surgery, Mount Sinai Saint Luke's, New York, NY; ${ }^{\mathrm{e} D e p a r t m e n t}$ of Thoracic and Cardiovascular Surgery, West German Heart and Vascular Center, University of Duisburg-Essen, Duisburg, Germany; ${ }^{\mathrm{f} D i v i s i o n}$ of Cardiothoracic Surgery, Veterans Affairs Medical Center, Washington, DC; and ${ }^{\mathrm{g}}$ Division of Cardiac Surgery, Libin Cardiovascular Institute of Alberta, University of Calgary, Calgary, Alberta, Canada.

The Registry for Quality Assessment with Ultrasound Imaging and Transit-time Flow Measurement in Cardiac Bypass Surgery study was funded by Medistim ASA, Oslo, Norway.
}

Read at the 99th Annual Meeting of The American Association for Thoracic Surgery, Toronto, Ontario, Canada, May 4-7, 2019.

Received for publication Jan 31, 2019; revisions received May 3, 2019; accepted for publication May 19, 2019; available ahead of print Nov 2, 2019.

Address for reprints: Stuart J. Head, MD, PhD, Department of Cardiothoracic Surgery, Erasmus MC, University Medical Center, Molewaterplein 40, 3015GD, Rotterdam, The Netherlands (E-mail: s.head@erasmusmc.nl).

0022-5223

Copyright (C) 2019 by The American Association for Thoracic Surgery. Published by Elsevier Inc. This is an open access article under the CC BY-NC-ND license (http:// creativecommons.org/licenses/by-nc-nd/4.0/).

https://doi.org/10.1016/j.jtcvs.2019.05.087 


\section{Abbreviations and Acronyms \\ $\mathrm{CABG}=$ coronary artery bypass grafting \\ HFUS = high frequency ultrasound \\ MACCE = major adverse cardiac and cerebrovascular event \\ MI $=$ myocardial infarction \\ REQUEST $=$ Registry for Quality Assessment with Ultrasound Imaging and Transit-Time Flow Measurement in Cardiac Bypass Surgery \\ TIA $=$ transient ischemic attack \\ TTFM $=$ transit-time flow measurements}

\section{A. Scanning this $Q R$ code will take \\ (4) you to the article title page to access supplementary informa- tion. To view the AATS Annual Meeting Webcast, see the URL next to the webcast thumbnail.}

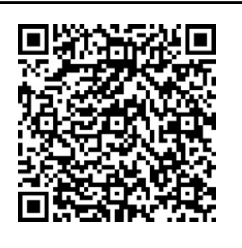

Since the introduction of coronary artery bypass grafting (CABG) more than 5 decades ago, surgeons have consistently focused on reducing perioperative adverse events and improving graft patency. Additionally, measures to improve long-term outcomes through superior conduit selection (more arterial grafts), implementation of less-invasive techniques and secondary preventive measures are constantly advocated. ${ }^{1,2}$ Although overall outcomes have significantly improved over time, the end points of mortality, stroke, and graft patency remain key targets for further optimization. ${ }^{3-5}$

Various intraoperative techniques continue to be recommended for contemporary state-of-the-art CABG. ${ }^{6,7}$ In terms of intraoperative quality assessment, efforts to improve surgical results have focused both on graft assessment by transit-time flow measurement (TTFM) and identification of disease-free areas of the aorta, using epiaortic high-frequency ultrasound (HFUS), to allow safe manipulation, and thereby reduce the risk of stroke. TTFM itself assesses graft flow and, by inference, the quality of the anastomosis and/or conduit and allows immediate graft revision in situations where the surgical result is not optimal.

A systematic review and meta-analysis of 6488 patients undergoing $\mathrm{CABG}$ reported that $4.3 \%$ of patients had graft revisions and that TTFM measurements correlated with graft patency during short- to midterm follow-up. ${ }^{8}$ In addition, HFUS is used for epiaortic scanning to identify diseased areas before any aortic manipulation, epicardial coronary artery scanning to identify potential target-vessel anastomosis sites that are free of plaque (especially on the posterior wall), ultrasound of the conduits when dissection is suspected, and postanastomosis scanning to identify potential anastomotic characteristics that limit graft flow.

The combination of these techniques, to avoid diseased areas of aorta and to identify and correct adverse technical issues affecting bypass grafts, could, intuitively, provide an important reduction in perioperative adverse events and improvement in graft patency. Indeed, Di Giammarco and colleagues $^{9}$ reported that the diagnostic accuracy of combining TTFM with HFUS on detecting truly patent or failing grafts increased significantly, reducing the proportion of unnecessary graft revisions.

The 2018 European Society of Cardiology/European Association for Cardio-Thoracic Surgery Guidelines on myocardial revascularization provide a IIa recommendation, Evidence Level B, for intraoperative graft flow assessment and Evidence Level $\mathrm{C}$ for epiaortic scanning during CABG. ${ }^{10}$ Nonetheless, published data on these techniques are limited to single-center studies and therefore its wider applicability to a broader cohort of patients in several centers is not immediately apparent. Consequently, the Registry for Quality Assessment with Ultrasound Imaging and Transit-Time Flow Measurement in Cardiac Bypass Surgery (REQUEST) study aims to determine the number and type of surgical procedure changes that are made based on intraoperative guidance information using the combination of TTFM and HFUS.

\section{METHODS \\ Study Design}

The REQUEST study is an international, multicenter, prospective registry that enrolled 1046 patients in 7 centers routinely performing CABG in Europe $(n=4)$ and North America $(n=3)$ between April 2015 and December 2017. This registry was designed to capture information on any changes in the proposed surgical procedure based on TTFM and HFUS parameters performed with the MiraQ or VeriQ C devices (Medistim ASA, Oslo, Norway).

All patients enrolled in the REQUEST study signed written informed consent before the surgical procedure. Each participating site received approval from their institutional review board or ethics committee before screening or enrollment of eligible patients.

The REQUEST study was funded by Medistim ASA (Oslo, Norway). The principal investigators and authors had complete scientific freedom.

The study is registered at ClinicalTrials.gov (NCT02385344).

\section{Study Protocol}

Participating surgeons and study coordinators at each site were trained to use and to interpret intraoperative TTFM and HFUS results according to a structured REQUEST study protocol. This protocol ensured that participating surgeons $(n=36)$ and personnel were trained in in the use of intraoperative TTFM and HFUS and possessed Good Clinical Practice certification. All participating surgeons had previous experience with using TTFM and HFUS and it was strongly recommended that surgeons had performed more than 20 cases with TTFM and HFUS before "participating in" the REQUEST study. 
The recommended surgical steps for quality assessment included HFUS assessment of the ascending aorta to the presence or absence of any plaques (soft and hard) or vessel wall abnormalities (eg, atheromatous ulcers and dissection) to establish the safest sites for cannulation, crossclamping, and side-biting for the proximal anastomosis; HFUS assessment of in situ conduits; HFUS assessment of target coronary vessels; and checking the conduit flow with TTFM and the anatomy of the constructed anastomosis with HFUS.

During intraoperative quality assessment, a steady mean arterial pressure of $80 \mathrm{~mm} \mathrm{Hg}$ was recommended and the HFUS probe frequency was set at $15 \mathrm{MHz}$. Furthermore, the REQUEST protocol advised, to standardize and optimize TTFM assessment, that the acoustic coupling index should be green or yellow (indicating the accuracy of ultrasound conductivity); the mean flow, indicated by the red line, should be steady and horizontal; the pulsatility index should be $<5$; the diastolic filling percent should be $>70 \%$ for left-sided and $>50 \%$ for right-sided coronary vessels.

It was at the discretion of the operating surgeon if and when a graft should be revised. If a graft was revised, it was recommended to reassess the conduit flow with TTFM and the anatomy of the reconstructed anastomosis with HFUS.

Although adherence to the study protocol was highly recommended it was not mandatory.

\section{Patient Population}

Patients diagnosed with multivessel coronary artery disease and scheduled for isolated CABG were eligible to be included. Patients were excluded from enrolment when undergoing emergency surgery, when concomitant surgical procedures were planned (eg, valve repair or replacement or rhythm surgery), when the medical history included the presence of a muscle disorder (eg, myopathy, myalgia, or myasthenia), or when the patient was known to be experiencing any psychological, developmental, or emotional disorder.

\section{End Points}

The primary end point was the overall frequency of any change in the planned surgical procedure as a consequence of TTFM, HFUS, and/or manual/visual assessment. Major secondary end points consisted of the rate and reason for any surgical change (eg, changes related to HFUS findings in the aorta, coronary targets, or in-situ conduits; and changes to completed grafts and/or anastomotic revision as a result of TTFM, HFUS, and/or manual/visual assessment). Additional end points consisted of the in-hospital rate of major adverse cardiac and cerebrovascular events (MACCE) defined as the composite of all-cause death, stroke or transient ischemic attack (TIA), myocardial infarction (MI), or repeat revascularization as well as the rates of the individual MACCE components and new hemodialysis and surgical exploration due to bleeding.

\section{Definitions}

A surgical change was defined by any change that was made intraoperatively by the operating surgeon based on TTFM, HFUS, and/or manual/visual assessment. Surgical changes related to the aorta due to the presence of calcification, plaques (soft or hard), and/or vessel wall abnormalities (ie, dissection) were divided into 3 groups: change in the proposed location of the cannulation site, the crossclamp site, and the site(s) for the proximal anastomosis (in a few cases this actually led to conversion of a planned on-pump procedure to an off-pump procedure with or without a no-touch aortic technique). Surgical changes associated with a coronary target were defined as changing the initially proposed location of the anastomosis due to severe calcification or plaque not identified by palpation (ie, usually on the posterior wall) or the detection of an intramural coronary artery that could not otherwise be found by visual assessment or manual palpation. Detailed definitions of surgical changes related to grafts and/or constructed anastomoses are shown in Table 1, along with relevant examples. In brief, surgical changes were categorized as a primary anastomotic revision, a secondary anastomotic revision, primary conduit revision, or an additional graft was used. Adding an extra suture for visual anastomotic bleeding was not classified as a surgical change. Any individual patient could have $\geq 1$ surgical change(s).

Stroke was defined as a sudden onset of focal neurological deficit of central origin that persisted for more than 24 hours with or without verification of the event on neuroimaging. TIA was defined as transient focal neurological signs or symptoms that lasted fewer than 24 hours. ${ }^{11}$ The diagnosis of perioperative MI was made based on clinical symptoms and additional cardiac biomarkers with or without abnormal electrocardiogram. New hemodialysis was defined as initiating renal replacement therapy in a patient with no prior history of renal replacement therapy, irrespective of the presence or absence of renal insufficiency at baseline. All clinical events were adjudicated by an otherwise blinded independent adjudicator. If consensus could not be reached, a second blinded adjudicator was consulted.

\section{Statistical Analyses}

Results are reported according to descriptive statistical methods. Categorical data are presented as proportions. Continuous data are reported as mean \pm standard deviation unless otherwise noted. Normality assumptions of the estimated proportions are based on a binomial distribution, funded on the central limit theorem and tested with the Kolmogorov-Smirnov test. Analyses were performed using SPSS software, version 24 (IBM-SPSS Inc, Armonk, NY).

\section{RESULTS}

\section{Baseline Characteristics}

The REQUEST study enrolled 1046 patients between April 2015 and December 2017. Of those, 30 patients were excluded due to screening failure $(n=8)$, members of the surgical team were not trained according to the REQUEST study protocol $(\mathrm{n}=11)$, or TTFM or HFUS images were unavailable for analysis $(n=11)$. The final analysis was performed on 1016 patients.

The mean age was 65.9 years and $14.0 \%$ (143 out of 1016) were women (Table 2). Diabetes mellitus was present in $39.6 \%$ of patients (402 out of 1016). A history of previous MI was recorded in $32.6 \%$ of patients (331 out of 1016 ) and $6.1 \%$ of patients (62 out of 1016) had a history of previous stroke. Multivessel disease was present in 981 patients $(96.6 \%)$ and 35 patients $(3.4 \%)$ had single vessel disease, which was a protocol violation.

\section{Surgical Characteristics}

The mean procedure time was 257 minutes (Table 3). Off-pump surgery was performed in $39.6 \%$ of cases (402 out of 1016). The mean number of conduits used per patient was 2.6 with a mean of 2.9 distal anastomoses per patient. Bilateral internal thoracic arteries were used in $30.5 \%$ (310 out of 1016), and $14.9 \%$ of grafts (398 out of 2675 ) were used in a Y or T configuration. Multiple arterial grafts were used in $43.2 \%$ of patients (439 out of 1016), with complete arterial revascularization being performed in $26.1 \%$ of cases (265 out of 1016 ). 
TABLE 1. Detailed definitions of surgical changes related to grafts with examples of surgical scenarios

\begin{tabular}{|c|c|c|}
\hline Surgical changes & Definition & Surgical examples \\
\hline $\begin{array}{l}\text { Primary anastomotic } \\
\text { revision }\end{array}$ & $\begin{array}{l}\text { Revision of the proximal or distal anastomosis due to a } \\
\text { primary technical problem with the anastomosis itself and } \\
\text { not due to a problem with the conduit }\end{array}$ & $\begin{array}{l}\text { i. Twisted anastomosis } \\
\text { ii. Removal of a stitch } \\
\text { iii. Redo of an anastomosis due to leaking, plaque, tapering, } \\
\text { bulging, a flap or narrowing } \\
\text { iv. Change from proximal anastomosis on the aorta to a } \\
\text { Y- or T-graft configuration } \\
\text { v. No other reason than poor flow }\end{array}$ \\
\hline $\begin{array}{l}\text { Secondary anastomotic } \\
\text { revision }\end{array}$ & $\begin{array}{l}\text { Revision of the proximal or distal anastomosis as a } \\
\text { consequence of needing to redo graft, but not due to a } \\
\text { problem with the anastomosis }\end{array}$ & $\begin{array}{l}\text { i. Kinked graft } \\
\text { ii. Inadequate length of the graft after the anastomosis } \\
\text { was completed (graft is shortened, ITA is used as } \\
\text { free graft, graft extended with additional graft) } \\
\text { iii. Dissected graft after the anastomosis was completed } \\
\text { iv. Change in graft configuration } \\
\text { v. Poor distal coronary bed }\end{array}$ \\
\hline $\begin{array}{l}\text { Primary conduit } \\
\text { revision }\end{array}$ & $\begin{array}{l}\text { Revision of the conduit without requiring revision of the } \\
\text { proximal or distal anastomosis }\end{array}$ & $\begin{array}{l}\text { i. Obstruction removed before completion of } \\
\text { the anastomosis (fibrin sealant removed) } \\
\text { ii. Inadequate conduit length before the anastomosis } \\
\text { was made } \\
\text { iii. Conduit extension based on visual assessment before } \\
\text { completion of the anastomosis } \\
\text { iv. Surgical clip is removed }\end{array}$ \\
\hline Additional graft(s) & $\begin{array}{l}\text { Need for an additional graft due to a problem with the } \\
\text { original anastomosis/conduit }\end{array}$ & $\begin{array}{l}\text { Persistent inadequate surgical results, despite primary } \\
\text { anastomotic revision, a secondary anastomotic revision, or } \\
\text { a primary graft revision }\end{array}$ \\
\hline
\end{tabular}

Detailed definitions of surgical changes used in the Registry for Quality Assessment study to classify surgical changes related to either the grafts or completed anastomoses. Illustrative surgical examples are provided to ensure better understanding of a situation during a coronary artery bypass procedure where a surgeon would use high frequency ultrasound or transit-time flow measurement to optimize patient and surgical outcomes. ITA, Internal thoracic artery.

Intraoperative quality assessment was performed in all 1016 patients. HFUS was used to assess the ascending aorta in $79.3 \%$ (806 out of 1016), the in situ conduits in $65.1 \%$ (661 out of 1016), the coronary targets in $47.5 \%$ (483 out of 1016) and completed grafts in 59.3\% (602 out of 1016). Moreover, patients who underwent off-pump surgery more frequently underwent HFUS of the ascending aorta $(88.3 \% ; 355$ out of 402) compared with patients who underwent on-pump surgery $(73.5 \% ; 451$ out of 614$)$. TTFM on grafts, with completed anastomoses, was performed in $99.2 \%$ (1008 out of 1016).

\section{Primary and Secondary End Points: Surgical Changes}

The primary end point of any surgical change was met in $25.2 \%$ of patients (256 out of 1016) (Table 4). In $77.0 \%$ (197 out of 256) this was the result of abnormal TTFM and/or HFUS findings, where on initial visual inspection there had been no suspicion of a diseased aorta, in situ conduit, coronary target, and/or completed graft. In $12.5 \%$ (32 out of 256 ) this was due to visual and/or manual assessment; for example, due to an obviously diseased aorta or a completed graft appeared unsatisfactory to the surgeon (eg, kinked or twisted graft). In $10.5 \%$ of patients ( 27 out of 256) it was undefined.

Surgical changes related to the aorta were made in $9.9 \%$ of patients ( 80 out of 806 ), and in 74 patients $(92.5 \%$ ) this was based on abnormal HFUS findings. This included changes related to the proposed locations of the cannulation site $(2.9 \% ; 23$ out of 806$)$, the crossclamp site $(2.2 \% ; 18$ out of 806), and the site for the proximal anastomosis $(6.8 \% ; 55$ out of 806$)$. HFUS detected the presence of soft plaques in the aorta in $9.1 \%$ of patients (73 out of 806 ) and calcification in $1.1 \%$ of patients ( 9 out of 806 ). The surgical strategy was changed from an on-pump to off-pump procedure in $0.2 \%$ of patients ( 2 out of 1016) due to plaques at the proposed cannulation or crossclamp site, and in $0.5 \%$ (5 out of 1016) proximal aortic anastomoses were avoided and patients received bilateral internal thoracic artery grafts or left internal thoracic artery-radial T-graft. On the contrary, the proposed procedure was changed from off-pump to on-pump in $0.7 \%$ (7 out of 1016) due to the extent of coronary artery disease and the concern that manipulation of the beating heart would cause hemodynamic instability. Among 80 patients with a surgical change related to the aorta, 59 underwent off-pump surgery $(73.8 \%)$. 
TABLE 2. Baseline characteristics of participants in the Registry for Quality Assessment with Ultrasound Imaging and Transit-Time Flow Measurement in Cardiac Bypass Surgery study

\begin{tabular}{lc}
\hline \multicolumn{1}{c}{ Characteristic } & Result \\
\hline Age (y) & $65.9 \pm 9.5$ y \\
$\geq 70$ & $37.3(379 / 1016)$ \\
Female sex & $14.0(143 / 1016)$ \\
\hline Body mass index, median (range) & $28.1(19-45)(1011 / 1015)$ \\
Diabetes mellitus & $39.6(402 / 1016)$ \\
Insulin treated & $17.4(177 / 1016)$ \\
\hline History of stroke & $6.1(62 / 1016)$ \\
History of myocardial infarction & $32.6(331 / 1016)$ \\
History of revascularization & $22.8(232 / 1016)$ \\
CABG & $0.7(7 / 1016)$ \\
PCI & $22.5(229 / 1016)$ \\
History of intervention for & $4.4(45 / 1016)$ \\
peripheral vascular disease & \\
Carotid & $2.7(27 / 1016)$ \\
Peripheral & $1.9(19 / 1016)$ \\
\hline LVEF (\%), median (range) & $51(17-82)(978 / 1016)$ \\
$<30$ & $2.5(24 / 978)$ \\
NYHA class & \\
I & $37.3(349 / 935)$ \\
II & $42.4(396 / 935)$ \\
III & $17.0(159 / 935)$ \\
IV & $3.3(31 / 935)$ \\
CCS angina classification & \\
I-II & $46.2(449 / 972)$ \\
III-IV & $41.5(403 / 972)$ \\
\hline Unstable angina & $30.7(312 / 1016)$ \\
Left main involvement & $54.8(437 / 797)$ \\
\hline Values are presented as mean \pm standard deviation, median (range), or $\%(\mathrm{n} / \mathrm{N})$ \\
LABG, Coronary artery bypass grafting; PCI, percutaneous coronary intervention; \\
CCS, Canadian Cardiovascular Society. & \\
&
\end{tabular}

Surgical changes related to in situ conduits occurred in $2.7 \%$ of patients (18 out of 661), and in 10 patients $(55.6 \%)$ this was based on abnormal HFUS and/or TTFM findings. In $0.6 \%$ (4 out of 661) this was due to dissection of the conduit and $0.8 \%$ (5 out of 661) of patients had conduits of insufficient caliber. In $1.4 \%$ (9 out of 661 ) the reason was unspecified.

In $22.6 \%$ (109 out of 483 ), a surgical change was made related to the proposed coronary target, and in 73 patients $(67.6 \%)$ this was the result of abnormal HFUS findings. This was due to a heavily diseased (ie, calcified) target in $10.6 \%$ of patients (51 out of 483 ), an intramural located coronary vessel in $3.9 \%$ (19 out of 483 ) and an insufficient caliber of the coronary target in $3.1 \%$ of patients ( 15 out of $483)$. Endarterectomies were performed in $1.2 \%$ of patients (6 out of 483). In $5.2 \%$ ( 25 out of 483 ) the reason for a
TABLE 3. Surgical characteristics of participants in the Registry for Quality Assessment with Ultrasound Imaging and Transit-Time Flow Measurement in Cardiac Bypass Surgery study

\begin{tabular}{lc}
\hline \multicolumn{1}{c}{ Surgical characteristic } & Result \\
\hline Procedure time* $(\mathrm{min})$ & $257 \pm 87$ \\
Off-pump surgery & $39.6(402 / 1016)$ \\
\hline LITA use & $96.8(983 / 1016)$ \\
BITA use & $30.5(310 / 1016)$ \\
\hline Radial artery use & $22.6(230 / 1016)$ \\
Multiarterial & $43.2(439 / 1016)$ \\
Complete arterial & $26.1(265 / 1016)$ \\
Y or T configuration-per graft & $14.9(398 / 2675)$ \\
Sequential grafts-per graft & $8.2(219 / 2675)$ \\
No. of conduits & \\
All & $2675(1015)$ \\
Per patient & $2.6 \pm 0.8(1015)$ \\
Arterial-per conduit & $58.3(1559 / 2675)$ \\
Venous-per conduit & $41.3(1105 / 2675)$ \\
Arteriovenous $\dagger-$ per conduit & $0.4(11 / 2675)$ \\
No. of distal anastomoses & \\
All & $2959(1015)$ \\
Per patient & $2.9 \pm 1.0(1015)$ \\
Arterial-per patient & $1.7 \pm 0.9(1015)$ \\
Venous-per patient & $1.2 \pm 1.0(1015)$ \\
\hline
\end{tabular}

Values are presented as mean \pm standard deviation, median (range), or \% $(\mathrm{n} / \mathrm{N})$. LITA, Left internal thoracic artery; BITA, bilateral internal thoracic arteries. *First incision to gloves off. $\dagger$ In cases where the arterial graft was too short to reach the coronary target, a venous graft was added.

surgical change related to the coronary target was unspecified.

Surgical changes related to grafts were performed in $7.8 \%$ of patients (79 out of 1016 ), including revisions to the proximal and/or distal anastomosis in $6.6 \%$ (67 out of 1016). In $4.1 \%$ of patients ( 42 out of 1016) this was based on a primary anastomotic revision. Surgical changes related to secondary anastomotic revisions occurred in $2.8 \%$ (28 out of 1016). In $64.5 \%$ of patients (51 out of 79) a surgical change related to a completed graft was the result of solely abnormal TTFM and/or HFUS findings (without visual and/or manual assessment), and of those $98.0 \%$ (50 out of 51) underwent repeat TTFM measurement after graft revision. In $90.0 \%$ (45 out of 50) the graft flow improved after graft revision.

Primary conduit revisions occurred in $1.5 \%$ ( 15 out of 1016 ) and in $0.5 \%$ of patients (5 out of 1016) an additional graft was needed. This resulted in any surgical revision for $3.4 \%$ of the anastomoses (100 out of 2959) Table E1.

\section{In-Hospital Clinical Outcomes}

The rate of in-hospital MACCE was 2.0\% (20 out of 1016) (Figure 1). In-hospital mortality was $0.6 \%$ (6 out of 1016). A stroke or TIA occurred in $1.0 \%$ (10 out of 
TABLE 4. Surgical changes in participants in the Registry for Quality Assessment with Ultrasound Imaging and Transit-Time Flow Measurement in Cardiac Bypass Surgery study

\begin{tabular}{|c|c|}
\hline Surgical change & Result \\
\hline \multicolumn{2}{|l|}{ Any surgical change } \\
\hline All changes in overall cohort & $25.2(256 / 1016)$ \\
\hline No. changes per patient (range) & $1(0-6)$ \\
\hline Change as a result of TTFM and/or HFUS & $77.0(197 / 256)$ \\
\hline Change as a result of visual/manual assessment & $12.5(32 / 256)$ \\
\hline Undefined & $10.5(27 / 256)$ \\
\hline \multicolumn{2}{|l|}{ Changes related to the aorta } \\
\hline All changes & $9.9(80 / 806)$ \\
\hline As a result of HFUS & $92.5(74 / 80)$ \\
\hline As a result of visual/manual assessment & $5.0(4 / 80)$ \\
\hline Undefined & $2.5(2 / 80)$ \\
\hline Cannulation site & $2.9(23 / 806)$ \\
\hline Crossclamp site & $2.2(18 / 806)$ \\
\hline Proximal anastomosis site & $6.8(55 / 806)$ \\
\hline \multicolumn{2}{|l|}{ Changes related to in situ conduits } \\
\hline All changes & $2.7(18 / 661)$ \\
\hline As a result of TTFM and/or HFUS & $55.6(10 / 18)$ \\
\hline As a result of visual/manual assessment & $33.3(6 / 18)$ \\
\hline Undefined & $11.1(2 / 18)$ \\
\hline Dissection & $0.6(4 / 661)$ \\
\hline Insufficient caliber & $0.8(5 / 661)$ \\
\hline \multicolumn{2}{|l|}{ Changes related to coronary target } \\
\hline All changes & $22.6(109 / 483)$ \\
\hline As a result of HFUS & $67.6(73 / 108)$ \\
\hline As a result of visual/manual assessment & $10.2(11 / 108)$ \\
\hline Undefined & $25.0(27 / 108)$ \\
\hline Diseased/calcified & $10.6(51 / 483)$ \\
\hline Detection of intramural vessel & $3.9(19 / 483)$ \\
\hline Insufficient caliber & $3.1(15 / 483)$ \\
\hline Endarterectomies & $1.2(6 / 483)$ \\
\hline \multicolumn{2}{|l|}{ Changes related to grafts } \\
\hline All changes & $7.8(79 / 1016)$ \\
\hline As a result of TTFM and/or HFUS & $64.5(51 / 79)$ \\
\hline As a result of visual/manual assessment & $34.2(27 / 79)$ \\
\hline Undefined & $7.6(6 / 79)$ \\
\hline Primary anastomotic revisions & $4.1(42 / 1016)$ \\
\hline Secondary anastomotic revisions & $2.8(28 / 1016)$ \\
\hline $\begin{array}{l}\text { Primary and secondary anastomotic } \\
\text { revisions combined }\end{array}$ & $6.6(67 / 1016)$ \\
\hline Primary graft revision & $1.5(15 / 1016)$ \\
\hline Additional graft(s) & $0.5(5 / 1016)$ \\
\hline
\end{tabular}

1016) and the rate of perioperative MI was $0.3 \%$ (3 out of 1016). One patient $(0.1 \%)$ underwent repeat revascularization by percutaneous coronary intervention. New hemodialysis occurred in $0.3 \%$ (3 out of 1016) and $0.2 \%$ ( 2 out of 1016) underwent surgical reexploration due to bleeding.

\section{DISCUSSION}

The REQUEST study is the first prospective, multicenter study that assessed the effects of routine use of TTFM with HFUS according to a prespecified protocol in patients with multivessel coronary artery disease undergoing CABG. As evidenced by the high proportion of off-pump operations and high use of multiple arterial grafts these procedures were performed by experienced CABG surgeons from 7 well-established institutions in Europe and North America. Overall, any surgical change to the initially proposed surgical strategy was made in $25.2 \%$ of patients, and in $77.0 \%$ this was solely based on otherwise unsuspected abnormal TTFM and/or HFUS findings. The present study showed that surgical changes related to the proposed site of aortic manipulation were made in $9.9 \%$ of patients and revisions to a completed graft in $7.8 \%$ of patients. Low rates of major adverse in-hospital events, and in particular mortality $(0.6 \%)$, indicate that intraoperative quality assessment of the aorta, the conduits, the coronary targets and completed grafts was safe (Figure 2).

Although intraoperative quality assessment of the aorta and grafts are both recommended by the 2018 European Society of Cardiology/European Association for Cardio-Thoracic Surgery Guidelines on myocardial revascularization, ${ }^{10}$ potential limitations of the TTFM technique, including uncertainty over its diagnostic accuracy, have been discussed. ${ }^{12}$ Furthermore, in the absence of clinical follow-up data, no clear consensus on the routine applicability of TTFM alone has been reached. Indeed, numerous single-center retrospective studies reported on the impact of adopting intraoperative quality assessment with TTFM, but this resulted in a substantial heterogeneity in definitions and outcomes. The overall proportion of revised grafts in relation to the number of patients studied ranged from $3.3 \%$ to $5.7 \%$ when TTFM alone was used. ${ }^{8}$ In the REQUEST study, surgical changes related to grafts occurred in $7.8 \%$ of patients. An important initiative in the REQUEST study was the routine use of HFUS in combination with TTFM during CABG. Di Giammarco and colleagues ${ }^{9}$ reported that the diagnostic accuracy of the combined use of TTFM and HFUS, reflected by the ability to detect a truly patent versus a truly failing graft (ie, positive predictive value vs negative predictive value), rose to $100 \%$ with this approach. The combination of TTFM with HFUS is therefore a potentially precise diagnostic tool for intraoperative quality assessment.

An additional important aspect of REQUEST is its contrast to previous retrospective studies reporting outcomes of intraoperative quality assessment that did not take into account the extent of surgeons' experience in using, interpreting and acting upon TTFM and HFUS findings. To address this specific issue, the REQUEST study 


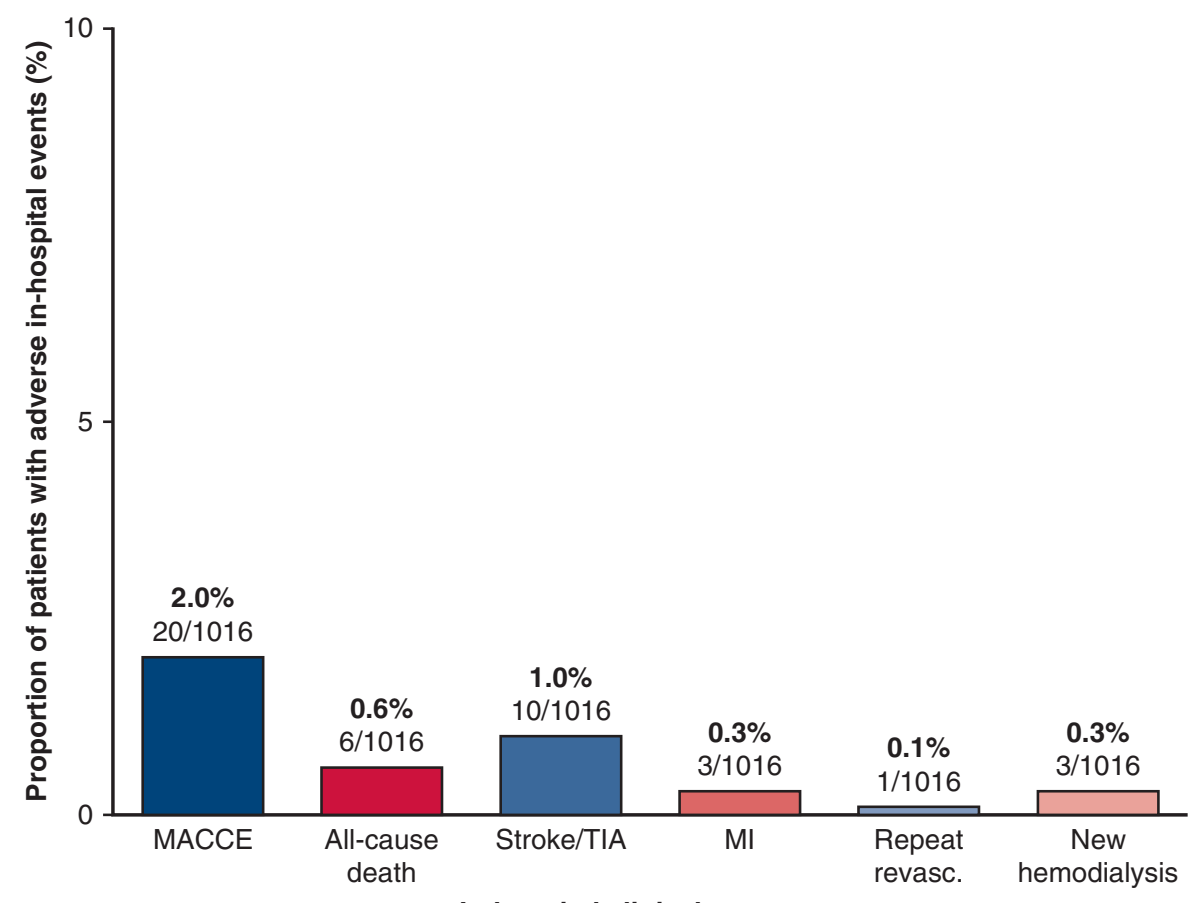

In-hospital clinical outcomes

FIGURE 1. In-hospital clinical outcomes in the Registry for Quality Assessment with Ultrasound Imaging and Transit-Time Flow Measurement in Cardiac Bypass Surgery study. Proportion (\%) of patients with in-hospital major adverse events (clinical events/number of observations). MACCE, Major adverse cardiac and cerebrovascular event; TIA, transient ischemic attack; $M I$, myocardial infarction.

was conducted in centers where the participating surgical team was trained according to the structured REQUEST protocol thereby minimizing bias in interoperator experience and interpretation of TTFM and HFUS findings.
To further enhance patient outcomes and optimize graft patency, intraoperative quality assessment of the surgical conduits and coronary targets was also performed in the REQUEST study. Changes made related to the conduits

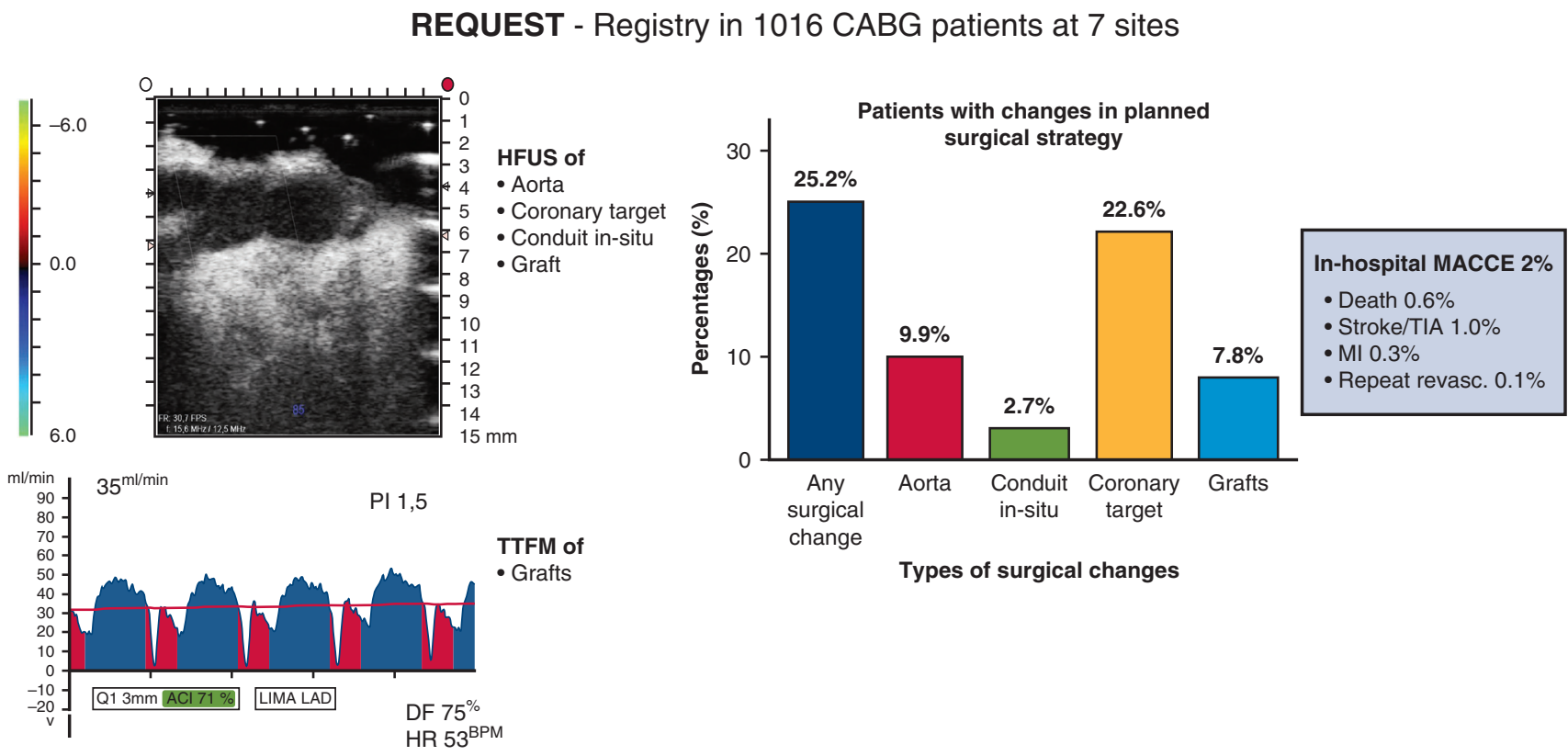

FIGURE 2. Changes to the planned surgical strategy were made in $25.2 \%$ of cases with few complications. CABG, Coronary artery bypass grafting; $H F U S$, High-frequency ultrasound; TTFM, transit-time flow measurement; MACCE, major adverse cardiac and cerebrovascular event; TIA, transient ischemic attack; $M I$, myocardial infarction. 
themselves were uncommon $(2.7 \%)$. Detection of an otherwise unrecognized dissected internal thoracic artery in some cases is likely to have prevented potential perioperative complications. In $10.6 \%$ of patients, a surgical change related to a heavily diseased and/or calcified coronary target was made. This may result in avoiding unnecessarily difficult anastomoses and/or endarterectomy. In only $1.2 \%$ of cases, an endarterectomy was performed, compared with the $4 \%$ endarterectomy rate in the Drug-Eluting Stent versus Coronary Artery Bypass Surgery for the Treatment of Narrowed Arteries study, ${ }^{13}$ and this could possibly be attributed to the use of epicardial HFUS to detect and avoid unsuitable targets for an anastomoses (typically a heavily diseased posterior wall). However, in patients with extensive coronary artery disease endarterectomy has shown to be an acceptable strategy to maximize completeness of revascularization during CABG. ${ }^{14,15}$

Because stroke is among the most devastating complications of CABG, avoiding any aortic manipulation is an important technique to reduce the rate of stroke. ${ }^{16-18}$ Previous studies report that during application and removal of the aortic crossclamp, particulate emboli are frequently detected. ${ }^{19-21}$ Aortic manipulation is often required for on-pump or off-pump surgery where a proximal anastomosis is made, although more than half of patients undergoing CABG have atherosclerosis in the ascending aorta with its inherent risk of embolism to the cerebral arteries. ${ }^{22}$ In the REQUEST study, off-pump CABG procedures were performed in $39.6 \%$. Despite the high rate of off-pump surgery, surgical changes related to the aorta were made in $9.9 \%$ of all patients to choose a safe place to cannulate, crossclamp, and/or construct the proximal anastomosis.

In the REQUEST study, although there was a very high use of TTFM (99\%) the use of HFUS was lower than expected. Although surgeons were advised to follow the REQUEST study protocol it was not deemed mandatory. The discrepancy in actual HFUS use most likely reflects real-world clinical decision making during coronary bypass surgery based on surgeon experience, preference, and discretion. For example, some situations in which some surgeons may decide that HFUS is not necessary might include:

- A proposed anastomotic site that looks and feels (eg, soft spot) completely normal,

- A conduit that looks completely normal after harvest, and

- A graft with excellent flow (eg, $>50 \mathrm{~mL} / \mathrm{min}$ with low pulsatility index) as indicated by TTFM.

Moreover, HFUS of the ascending was more frequently performed during off-pump surgery $(88 \% ; 355$ out of 402) compared with on-pump surgery $(73 \% ; 451$ out of 614). Additionally, patients with a surgical change related to the aorta $(\mathrm{n}=80)$ were more often patients who underwent off-pump surgery $(74 \% ; \mathrm{n}=59)$ compared with on-pump surgery $(26 \% ; n=21)$. The surgical changes to the aorta in the off-pump cohort were mainly related to determining the optimal (ie, disease free) location for the proximal anastomosis to diminish the detrimental effects of aortic manipulation in patients with an increased atherosclerotic burden.

Finally, while HFUS is relatively easy to use for grafts to the anterior wall, it is technically more challenging to use and to interpret for grafts on the lateral and inferior wall, especially in off-pump procedures. Therefore, the actual result of HFUS on surgical decision making and clinical outcomes might have been even greater if it had been used in all patients. However, compared with other contemporary studies there was no significant reduction in cerebrovascular events (eg, $1.0 \%$ stroke/TIA in the current study), which could have been the result of the high rate of patients with severe atherosclerotic disease (eg, diabetes was present in almost $40 \%$ ) and the fact that epiaortic scanning was performed in $79 \%$ of patients.

\section{Limitations}

Although REQUEST is currently the largest prospective study to evaluate the influence of routine intraoperative quality assessment in contemporary CABG, its most obvious limitation is that it was not a randomized trial and therefore did not compare CABG with versus without use of TTFM and HFUS. The reality is that the design of such a randomized controlled trial would need an extraordinarily large patient sample size (eg, to demonstrate a reduction in operative mortality from $1 \%-0.5 \%$ ) and extensive long-term follow-up to assess the influence of improved long-term graft patency. Furthermore, interpretation of such a trial would be complicated by a myriad of other factors that influence short- and long-term outcomes of CABG.

The focus of the REQUEST study was to prospectively evaluate the impact of TTFM with HFUS on patients who underwent $\mathrm{CABG}$ procedure, when performed routinely by experienced CABG surgeons. Although, as explained above, conducting an randomized controlled trial would be extremely challenging for several reasons. A further limitation is that no conclusions can be drawn on the influence of intraoperative quality assessment on long-term outcomes because clinical results were limited to in-hospital outcomes. Similarly, in the absence of long-term angiographic follow-up, no inference can be drawn on whether routine graft assessment actually improves long-term graft patency. Finally, use of TTFM and HFUS technology requires dedicated training to use the technology appropriately and to both correctly interpret data and avoid the pitfalls of misinterpretation. 


\section{CONCLUSIONS}

The REQUEST study is a multicenter, prospective study designed to evaluate the effect of implementing intraoperative quality assessment by TTFM and HFUS in 1016 patients undergoing CABG. In $25.2 \%$ of patients, any change in the planned surgical strategy was made. Furthermore, rates of in-hospital mortality and major morbidity were low, showing the feasibility and safety of performing intraoperative quality assessment. To conclude, intraoperative quality assessment with TTFM and HFUS may improve the quality, safety, and efficacy of CABG procedures and should therefore be considered as a routine procedural aspect during $\mathrm{CABG}$.

\section{Webcast}

You can watch a Webcast of this AATS meeting presentation by going to: https://aats.blob.core.windows. net/media/19\%20AM/Sunday_May5/206BD/206BD/S52\% 20-\%20CABG \%20Technical \% 20Considerations/S52_5 webcast_023136427.mp4.

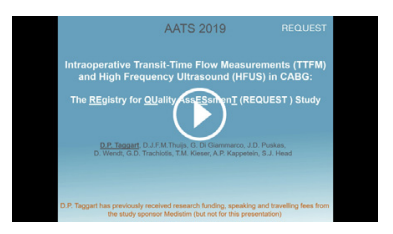

\section{Conflict of Interest Statement}

Dr Taggart has received research funding, speaking, and traveling honoraria from Medistim. Drs Thuijs, Trachiotis, Puskas, Wendt, Kieser, Kappetein, and Head received traveling support and/or speaking fees from Medistim. Drs Kappetein and Head are full-time employees of Medtronic outside the submitted work. All other authors have nothing to disclose with regard to commercial support.

The authors thank the study coordinators at the 7 participating study sites. Finally, the authors thank Professor Vipin Zamvar and Yasir Abu-Omar for independently adjudicating the serious adverse events in the REQUEST study.

\section{References}

1. Head SJ, Kieser TM, Falk V, Huysmans HA, Kappetein AP. Coronary artery bypass grafting: part 1-the evolution over the first 50 years. Eur Heart J. 2013;34:2862-72.

2. Head SJ, Borgermann J, Osnabrugge RL, Kieser TM, Falk V, Taggart DP, et al. Coronary artery bypass grafting: part 2-optimizing outcomes and future prospects. Eur Heart J. 2013;34:2873-86.

3. Gaudino M, Antoniades C, Benedetto U, Deb S, Di Franco A, Di Giammarco G, et al. Mechanisms, consequences, and prevention of coronary graft failure. Circulation. 2017;136:1749-64.

4. Thielmann M, Massoudy P, Jaeger BR, Neuhäuser M, Marggraf G, Sack S, et al. Emergency re-revascularization with percutaneous coronary intervention, reoperation, or conservative treatment in patients with acute perioperative graft failure following coronary artery bypass surgery. Eur J Cardiothorac Surg. 2006;30:117-25.
5. Dacey LJ, Likosky DS, Leavitt BJ, Lahey SJ, Quinn RD, Hernandez F Jr, et al Perioperative stroke and long-term survival after coronary bypass graft surgery. Ann Thorac Surg. 2005;79:532-6.

6. Kowalewski M, Pawliszak W, Malvindi PG, Bokszanski MP, Perlinski D, Raffa GM, et al. Off-pump coronary artery bypass grafting improves short-term outcomes in high-risk patients compared with on-pump coronary artery bypass grafting: meta-analysis. J Thorac Cardiovasc Surg. 2016;151: 60-77.

7. Gaudino M, Bakaeen F, Davierwala P, Di Franco A, Fremes SE, Patel N, et al. New strategies for surgical myocardial revascularization. Circulation. 2018; 138:2160-8.

8. Thuijs DJFM, Bekker MWA, Taggart DP, Kappetein AP, Kieser TM, Wendt D, et al. Improving coronary artery bypass grafting: a systematic review and meta-analysis on the impact of adopting transit-time flow measurement. Eur $J$ Cardiothorac Surg. 2019;56:654-63.

9. Di Giammarco G, Canosa C, Foschi M, Rabozzi R, Marinelli D, Masuyama S, et al. Intraoperative graft verification in coronary surgery: increased diagnostic accuracy adding high-resolution epicardial ultrasonography to transit-time flow measurement. Eur J Cardiothorac Surg. 2014;45:e41-5.

10. Sousa-Uva M, Neumann F-J, Ahlsson A, Alfonso F, Banning AP, Benedetto U, et al. 2018 ESC/EACTS guidelines on myocardial revascularization. Eur J Cardiothorac Surg. 2019;55:4-90.

11. Lansky AJ, Messé SR, Brickman AM, Dwyer M, Bart van der Worp H, Lazar RM, et al. Proposed standardized neurological endpoints for cardiovascular clinical trials: an Academic Research Consortium initiative. $J$ Am Coll Cardiol. 2017;69:679-91.

12. Niclauss L. Techniques and standards in intraoperative graft verification by transit time flow measurement after coronary artery bypass graft surgery: a critical review. Eur J Cardiothorac Surg. 2017;51:26-33.

13. Head SJ, Parasca CA, Mack MJ, Mohr FW, Morice MC, Holmes DR Jr, et al Differences in baseline characteristics, practice patterns and clinical outcomes in contemporary coronary artery bypass grafting in the United States and Europe: insights from the SYNTAX randomized trial and registry. Eur J Cardiothorac Surg. 2015;47:685-95.

14. LaPar DJ, Anvari F, Irvine JN Jr, Kern JA, Swenson BR, Kron IL, Ailawadi G. The impact of coronary artery endarterectomy on outcomes during coronary artery bypass grafting. J Card Surg. 2011;26:247-53.

15. Garcia S, Sandoval Y, Roukoz H, Adabag S, Canoniero M, Yannopoulos D, et al Outcomes after complete versus incomplete revascularization of patients with multivessel coronary artery disease: a meta-analysis of 89,883 patients enrolled in randomized clinical trials and observational studies. J Am Coll Cardiol. 2013; 62:1421-31

16. Head SJ, Milojevic M, Daemen J, Ahn JM, Boersma E, Christiansen EH, et al Stroke rates following surgical versus percutaneous coronary revascularization. J Am Coll Cardiol. 2018;72:386-98.

17. Rajsic S, Gothe H, Borba HH, Sroczynski G, Vujicic J, Toell T, et al. Economic burden of stroke: a systematic review on post-stroke care. Eur J Health Econ 2019;20:107-34.

18. Zhao DF, Edelman JJ, Seco M, Bannon PG, Wilson MK, Byrom MJ, et al. Coronary artery bypass grafting with and without manipulation of the ascending aorta: a network meta-analysis. J Am Coll Cardiol. 2017;69: 924-36.

19. Banbury MK, Kouchoukos NT, Allen KB, Slaughter MS, Weissman NJ Berry GJ, et al. Emboli capture using the Embol-X intraaortic filter in cardiac surgery: a multicentered randomized trial of 1,289 patients. Ann Thorac Surg. 2003; 76:508-15.

20. Christenson JT, Vala DL, Licker M, Sierra J, Kalangos A. Intra-aortic filtration: capturing particulate emboli during aortic cross-clamping. Tex Heart Inst J. 2005;32:515-21.

21. Gerriets T, Schwarz N, Sammer G, Baehr J, Stolz E, Kaps M, et al. Protecting the brain from gaseous and solid micro-emboli during coronary artery bypass grafting: a randomized controlled trial. Eur Heart J. 2010;31:360-8.

22. Suvarna S, Smith A, Stygall J, Kolvecar S, Walesby R, Harrison M, et al An intraoperative assessment of the ascending aorta: a comparison of digital palpation, transesophageal echocardiography, and epiaortic ultrasonography. J Cardiothorac Vasc Anesth. 2007;21:805-9.

Key Words: coronary artery bypass grafting, transit time, high frequency ultrasound, intraoperative quality control, REQUEST 


\section{Discussion}

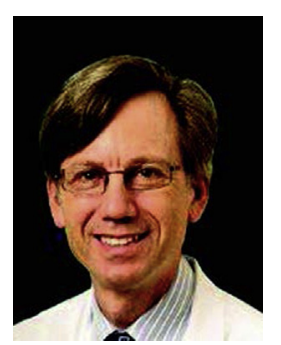

Dr Stephen E. Fremes (Toronto, Ontario, Canada). Thanks for the privilege of discussing this paper. David, I want to congratulate you on a very important study and excellent presentation and for providing me with the manuscript and slides well in advance.

I concur with your conclusions. I have a few questions. My disclosures: I do use transit-time flow measurement regularly; I use high-frequency ultrasound less frequently, more selectively. And I will give you a question and you can answer me, and if you want to editorialize as well as just answer, that is your prerogative.

Was there a protocol for mandated graft revisions or was this according to the surgeon's decision in concert with the findings?

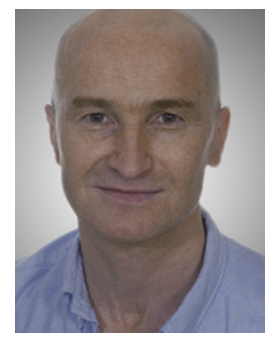

Dr David Paul Taggart (Oxford, United Kingdom). We left this essentially to surgeon experience, discretion, and preference, because these were experienced surgeons. We thought about having mandated changes, but it's actually very difficult to know, because if you take into consideration flow and pulsatility and things you have to think about, competitive flow, we decided to leave it in the hands of surgeons who had been trained to use this technology.

Dr Fremes. Could you quantify the number of patients who did and didn't get revised against some standard criteria?

Dr Taggart. No, and, again, this is a problem of an observational rather than a randomized trial. What we saw was that even in the hands of experienced surgeons, in $25 \%$ of the patients they made some changes to what they had initially proposed to do.

Dr Fremes. Was there any evidence of a learning curve during the trial either by better adherence to the protocol or a difference in the number of revisions as the trial progressed?

Dr Taggart. The answer to that is both yes and no. What I mean by that is right from the outset, $99 \%$ of patients had transit-time flow measurement. So from the start, every surgeon agreed we want to know the flow in the grafts. But the use of the ultrasound was more variable. So it was quite high for the ascending aorta, 90\%, but initially it was lower for imaging coronary targets, harvested conduits and the anastomosis itself. But definitely as the study progressed and people became more familiar with the use of it, that increased.

Dr Fremes. When do you evaluate the distal anastomosis during on-pump surgery?

Dr Taggart. For distal anastomosis on-pump, the easiest, most convincing, and best to do is the left internal thoracic artery to the left anterior descending artery with the crossclamp still on. You take the bulldog off the internal thoracic artery and you then measure the flow in that when there is no competitive flow whatsoever, and the highest volume we have seen for an internal thoracic artery to the left anterior descending artery with a crossclamp is $440 \mathrm{~mL} / \mathrm{min}$. As soon as you open the clamp, it drops by $50 \%$. And for the other grafts, if they are vein grafts or others, if there are conduits inside you would do the same; if it's vein grafts, then you have to wait until you have completed the top end and while the patient is still on bypass.

Dr Fremes. Were the images reviewed centrally, and if so, what was the correlation?

Dr Taggart. No, there was no central review. Assessment of the images were made within individual centers.

Dr Fremes. One of the authors, di Giammarco, has previously reported on false positives and false negatives with transit-time flow measurement and the high-frequency ultrasound was sort of the gold standard. Can you comment on that previous finding and this study?

Dr Taggart. I think it's a great question, and looking back we did not mandate to do that, but perhaps in retrospect we should have done it. But if you see the figures of the actual use of ultrasound assessment of the anastomosis, it was only about $56 \%$. And as a confirmed off-pump surgeon, I can usually get a good image of the internal thoracic artery to the left anterior descending artery, and that satisfies me, because that's where you have the biggest bang for the buck, but doing off-pump to lateral and inferior wall is more challenging. 
TABLE E1. Proportion of surgical changes related to grafts, per distal anastomosis, as a result of abnormal transit-time flow measurement (TTFM) and/or high-frequency ultrasound (HFUS) findings in the Registry for Quality Assessment with Ultrasound Imaging and Transit-Time Flow Measurement in Cardiac Bypass Surgery study

\begin{tabular}{ll}
\hline \multicolumn{1}{c}{ Surgical change } & \multicolumn{1}{c}{ Result } \\
\hline Changes related to grafts & \\
All changes & $3.4(100 / 2959)$ \\
Primary anastomotic revisions & $1.6(48 / 2959)$ \\
Secondary anastomotic revisions & $1.0(31 / 2959)$ \\
Primary graft revision & $0.5(16 / 2959)$ \\
Additional graft(s) & $0.2(5 / 2959)$ \\
\hline
\end{tabular}

Values are presented as $\%(\mathrm{n} / \mathrm{N})$. 
TABLE E1. Proportion of surgical changes related to grafts, per distal anastomosis, as a result of abnormal transit-time flow measurement (TTFM) and/or high-frequency ultrasound (HFUS) findings in the Registry for Quality Assessment with Ultrasound Imaging and Transit-Time Flow Measurement in Cardiac Bypass Surgery study

\begin{tabular}{ll}
\hline \multicolumn{1}{c}{ Surgical change } & \multicolumn{1}{c}{ Result } \\
\hline Changes related to grafts & \\
All changes & $3.4(100 / 2959)$ \\
Primary anastomotic revisions & $1.6(48 / 2959)$ \\
Secondary anastomotic revisions & $1.0(31 / 2959)$ \\
Primary graft revision & $0.5(16 / 2959)$ \\
Additional graft(s) & $0.2(5 / 2959)$ \\
\hline
\end{tabular}

Values are presented as $\%(\mathrm{n} / \mathrm{N})$. 